\title{
Advanced considerations in organ donors
}

\author{
Hailey M. Shepherd ${ }^{1}$, Jason M. Gauthier ${ }^{1}$, Varun Puri ${ }^{1}$, Daniel Kreisel ${ }^{1,2}$, Ruben G. Nava ${ }^{1}$ \\ ${ }^{1}$ Division of Cardiothoracic Surgery, Department of Surgery, Washington University School of Medicine, Saint Louis, MO, USA; ${ }^{2}$ Department of \\ Pathology and Immunology, Washington University School of Medicine, Saint Louis, MO, USA \\ Contributions: (I) Conception and design: All authors; (II) Administrative support: V Puri, D Kreisel, RG Nava; (III) Provision of study materials \\ or patients: V Puri, D Kreisel, RG Nava; (IV) Collection and assembly of data: HM Shepherd, JM Gauthier, RG Nava; (V) Data analysis and \\ interpretation: All authors; (VI) Manuscript writing: All authors; (VII) Final approval of manuscript: All authors. \\ Correspondence to: Ruben G. Nava, MD. Division of Cardiothoracic Surgery, Washington University School of Medicine, Campus Box 8109 , 660 \\ South Euclid Avenue, St. Louis, MO 63110, USA. Email: navar@wustl.edu.
}

\begin{abstract}
The rising need for lung transplantation over recent years has not paralleled the availability of suitable lung allografts. The number of lung transplantations performed each year in the United States remains limited by an inadequate supply of suitable donors as well as low donor utilization rates. While several methods have been proposed for increasing the donor pool, there is considerable disparity between acceptance and utilization of these practices among transplant centers. In this review article, we explore various approaches for enhancing donor selection and expanding the donor pool. We discuss the use of "extended criteria" donors including high risk groups such as drug overdose donors, and we examine the role of techniques in donor assessment and selection such as the use of computed tomography for accurate size matching. We review topics in donor management such as the establishment of specialized donor care facilities and the implementation of lung-focused resuscitation protocols, and we discuss advancements in donor procurement such as the utilization of local procurement teams. We also review barriers to donation, such as variability in organ procurement organization (OPO) consent practices, as well as patient-specific factors such as religious or cultural beliefs. Addressing these aspects of donor evaluation, management, and accessibility is essential in maximizing the number of lungs available for transplantation within the existing donor pool.
\end{abstract}

Keywords: Lung transplant; organ donor

Submitted Jun 29, 2020. Accepted for publication Feb 02, 2021.

doi: 10.21037/jtd-2021-08

View this article at: http://dx.doi.org/10.21037/jtd-2021-08

\section{Introduction}

Nearly 1,500 candidates remained on the lung transplant waiting list at the end of 2018, a figure which has remained largely stable over the last decade (1). While the U.S. has answered the demand for lung transplantation with an increase in annual lung transplants, the number of patients added to the waitlist has also increased in recent years. As such, it appears that the increase in supply has been met with an increase in demand, and there remains an inadequate supply of suitable donor lungs. Compounding the shortage of donor lungs is low donor utilization rates, with only $20 \%$ of eligible lungs from potential donors being transplanted (2). Given these statistics, it is imperative to explore innovative options that increase the availability of donor lungs in a manner which does not adversely impact allograft survival.

Several novel methods for increasing the donor pool have been suggested, such as lobar lung transplantation, cardiac death donors, and ex-vivo lung perfusion. Until these measures can be widely adopted across transplant centers, the most practical means of increasing lung transplants is to maximize use of the existing donor pool. In recent years, several novel facets of the donor evaluation have emerged which may influence lung transplant rates. In this article, we discuss several of these advanced considerations in 
organ donors. In is our hope that this review will provide transplant providers with a better understanding of these components of the donor evaluation and allow for a safe increase in donor lung utilization.

\section{Selection of lung donors}

\section{Extended criteria lung donors}

Historical donor selection criteria for "ideal" lungs were largely constructed from clinical impressions rather than data-driven evidence (3-5). The components of ideal lung donor criteria (including age $\leq 55, \mathrm{ABO}$ compatibility, clear chest $\mathrm{X}$-ray, ratio of arterial oxygen partial pressure to fraction of inspired oxygen $(\mathrm{P} / \mathrm{F}$ ratio) $>300$, less than 20 pack-year smoking history, lack of chest trauma or prior cardiopulmonary surgery, negative sputum gram stain, and bronchoscopy without purulent secretions) have been studied individually with many showing some association to recipient outcomes. However, the ideal lung donor criteria collectively remain unsubstantiated by prospective controlled trials or consistent outcomes data $(6,7)$. Adherence to strict donor guidelines appears to result in a relatively low utilization of potential lung donors, with only $20 \%$ of eligible lungs from multi-organ donors undergoing transplantation (2). Meanwhile, the 1-year mortality of patients awaiting lung transplant approaches $20 \%$, and the waitlist length has remained steady over the last decade despite an increase in total lung transplants annually (8). Given the increasing demand and inadequate supply of donor lungs, identifying strategies for safely increasing the donor pool is imperative.

In recent years, many institutions have liberalized their donor criteria by accepting "marginal" or "extended criteria" donors, which do not meet traditional "ideal" donor criteria. In 2012, a landmark article from Hannover demonstrated equivalent recipient survival when transplanted with lungs which had been previously turned down by at least three centers, compared to standard criteria donor lungs (9). In 2019, authors re-demonstrated similar results in the pediatric population (10). Other studies analyzing outcomes of rejected lungs are limited, and it remains to be determined whether the results of the Hannover group are generalizable to other institutions. Notably, a recent study from the U.S. which employed OPTN/UNOS data from nearly 11,000 lung transplants found a higher hazard of death among recipients receiving extended criteria donor lungs. This finding was significant in both standard risk (lung allocation score $<70$ ) and high risk lung recipients (LAS $\geq 70$ ), with high risk recipients having a hazard ratio of 1.8 when extended criteria donor lungs were used (11). While such findings from the U.S. are difficult to compare to those from Hannover for several reasons, the discrepancy highlights that caution must be used when deviating from ideal lung donor criteria until additional studies emerge.

A study using UNOS data has identified a subset of "ideal" lung donor criteria that do not appear to significantly impact recipient survival (i.e., P/F ratio, abnormal chest $\mathrm{x}$-ray, purulent secretions on bronchoscopy), while others were consistently associated with adverse outcomes (i.e., smoking history). In this study, more than half of the transplanted lungs possessed at least one variable that did not meet ideal lung donor guidelines. Importantly, this study also identified novel donor variables associated with increased recipient mortality, such as diabetes, cytomegalovirus immunity, and blood type A, which are not part of the traditional lung donor guidelines (12). Another analysis of the UNOS database determined that poor donor $\mathrm{PaO} 2$ was not associated with reduced graft survival. Interestingly, the percent of donor lungs with $\mathrm{PaO} 2$ less than $200 \mathrm{mmHg}$ increased over time, comprising $5 \%$ of transplants in 2000 and $21 \%$ of transplants by 2009 (13). These results suggest that many centers are beginning to transplant lungs which do not meet ideal criteria. The literature collectively suggests that presence of one or two suboptimal variables in an extended criteria donor are likely associated with acceptable outcomes, but the threshold for allowing suboptimal donor variables remains unknown. Revision of current guidelines with validated donor criteria is essential. With clarification of meaningful measures of lung quality with randomized multi-institutional trials, the supply of donor lungs can be safely expanded without impacting post-transplant survival. Until this is achieved, we recommend judicious use of donor lungs which do not meet traditional donor criteria.

\section{Drug overdose donors}

Unintentional injury accounts for the highest proportion of deaths in individuals under 45 years old, with an alarming number attributed to drug overdose (14). Drug overdose disproportionately affects young and middle-aged adults who possess less comorbidities compared to older adults $(15,16)$. Despite this, fewer organs are transplanted from drug overdose donors when compared to those with other causes of death (16). This discrepancy is thought to be 
largely out of concern for blood-borne disease transmission $(17,18)$. Fortunately, recent evidence has demonstrated that despite increased utilization of organs from donors after death from drug overdose, recipient outcomes are comparable. A recent study in the Annals of Thoracic Surgery similarly found that hearts transplanted from drug overdose donors had drastically increased in recent years and were associated with equivalent survival outcomes when compared to other causes of death. However, drug overdose heart donors were more likely to be hepatitis $\mathrm{C}$ positive in this study (19). Until more data becomes available, there must be an open and honest discussion between the surgeon and potential recipient regarding the uncertain risks associated with these organ donors. We are hopeful it will soon be established that utilizing lungs from drug overdose donors is a safe, novel method to expand the donor pool.

\section{Chest CT imaging}

While CT imaging was not traditionally a part of the donor lung evaluation, surgeons are often presented with chest CT findings at the time of an organ offer. The impact of such findings often creates a dilemma for transplant surgeons, as there is little data available to guide their decision of whether or not to accept lungs. Certain donor CT findings obviously prohibit donor lungs from transplantation, such malignancy or widespread ground-glass opacities consistent with acute-respiratory distress syndrome. However, reversible findings such as traumatic lung injury or pneumonia may be compatible with favorable recipient outcomes.

To better understand the utility of donor chest CT scans in lung donor assessment, we recently conducted a retrospective analysis of all brain death organ donors from our local organ procurement organization (OPO) over a 5 -year period ( $\mathrm{N}=753)$. We found that $59 \%$ of potential lung donors had received a CT scan by the time of organ harvest, revealing numerous types of pathology such as pneumonia (32\%), aspiration (20\%), and traumatic lung injury (7\%), among others. While the presence of a chest CT scan was not associated with increased odds of lung acceptance, specific findings were associated with a change in the odds of acceptance. Most notably, in a multivariate analysis, findings of structural lung disease, including both emphysema and interstitial lung disease, were associated with decreased odds of lung acceptance. Interestingly, several case studies from this data set revealed patients with acceptable donor variables and adequate oxygenation, but CT findings of structural lung disease, and these lungs were declined. These results demonstrate that donor chest CT scans are influencing the decision to accept donor lungs (20). Future studies which translate such donor CT scans to recipient outcomes are warranted and in process at this time.

Another benefit of chest CT imaging is that it allows for more accurate measurement of donor lungs, compared to chest X-ray or total lung capacity, so lungs may be appropriately size-matched to the potential recipient. Donor-recipient mismatch has been previously shown to be associated with both early and late graft dysfunction $(21,22)$. CT imaging may also serve as a method of remotely evaluating of donor lungs to reduce "negative" fly-outs, in which a procurement team travels to an outside hospital but determines the lungs to be unsuitable for their recipient during the intra-op evaluation. If a donor's lungs can be accurately vetted for pathology or size mismatch based on CT images, the resource intensive process of sending a procurement team to an outside hospital for evaluation may be avoided.

\section{Management}

\section{Specialized donor care facility (SDCF)}

In 2001, our OPO built the nation's first SDCF in St. Louis, Missouri. In this model, donation after brain death (DBD) organ donors are transferred to the freestanding facility for management after consent for donation is obtained. The SDCF houses an intensive care unit (ICU), staffed with critical care nurses and intensive care physicians, which manages the donor until the time of organ procurement. The SDCF has onsite resources for laboratory workup including HLA typing, imaging (i.e., CT scanner), and invasive procedures (i.e., bronchoscopy, echocardiography and catheterization), which can be obtained on request from transplant centers. Once the organ allocation process is complete, transplant teams then travel to SDCF for organ procurement, which occurs in one of the SDCF's operating rooms. Since the development of this model, 4 other OPOs have developed a SDCF and 7 others are in the process of doing so.

The SDCF model has changed donor management in several ways. A study from our institution found that SDCF procurement reduced surgeon travel time from 8 to 2.7 hours with a $93 \%$ reduction in air travel (23). Furthermore, SDCF procurement led to significantly higher organ yield for both standard criteria donors (3.92 vs. 3.7 per donor) and 
extended criteria donors (2.2 vs. 1.87 per donor) (24). Other organ procurement centers have also shown similar promise in facilitating donor procurements and increasing donor availability (25). While outside the scope of this article, the SDCF model appears to be a cost-effective means of donor management, and it is likely that more OPOs will adopt this model of care in the coming decade $(23,24)$.

Since the SDCF model is becoming increasingly common, it warrants attention from transplant centers. By having a potential organ donor residing in such a facility, there lies the opportunity for a more focused, efficient donor workup and procurement process. The SDCF model also facilitates lung focused donor resuscitation, as discussed below, which is difficult to accomplish in the traditional model of hospital-based donor care.

\section{Lung focused resuscitation protocols}

The clinical management of lung donors can be a challenging process for the hospital/SDCF where the donor resides. In a multi-organ donor, a fluid balance that may favor preservation of kidneys may adversely impact the suitability of a donor's lungs for transplantation. As such, it is imperative that organ focused resuscitation protocols have an established impact on organ utilization so that such measures do not needlessly compromise other organs in the same donor.

Since our OPO employs the SDCF model, we have had the unique opportunity to study lung focused resuscitation protocols to determine their impact on lung utilization. In 2008, the SDCF implemented a protocol whereby multi-organ donors are managed with specific ventilator strategies (i.e., tidal volume $6-8 \mathrm{~mL} / \mathrm{kg}$, I:E ratio of $1: 1$, PEEP $8-10 \mathrm{cmH}_{2} \mathrm{O}$, etc.), early digital bronchoscopy, serial chest $\mathrm{X}$-rays, CT scan if the donor is deemed suitable for lung transplantation, and a specific medical regimen (i.e., hydrocortisone), among others. The organ utilization rate was then analyzed in donors prior to $(\mathrm{N}=791)$ and after $(\mathrm{N}=1,333)$ implementation of the lung focused resuscitation protocol. We found that the mean organ yields significantly increased from 3.5 to 3.8 organs per donor after implementation of the lung focused resuscitation protocol. Notably, the lung utilization rate increased substantially from $19.8 \%$ to $33.9 \%$ after implementation (8). These results not only established a sound lung focused resuscitation protocol, but perhaps more importantly, also showed that such a protocol does not lead to decreased utilization of other organs. While such interventions may be difficult to accomplish outside of a SDCF setting, we encourage lung transplant centers to request these interventions from hospitals managing potential lung donors.

\section{Lung procurement and transport}

Transportation plays a pivotal role in augmenting geographic disparities and ischemia time with lung donor procurement. After the new allocation policy instituted in 2017, some transplant centers have noticed a decline in local lung transplants with increasing distant organ procurements which require air travel (26). A landmark survey conducted in 2007 was administered to transplant surgeons to investigate travel trends during organ procurement, which revealed an average travel distance of 300 nautical miles for lung retrieval, despite the closest transplant center being located most commonly within 50 miles of the donor hospital. More alarming data which emerged from the survey demonstrated that $80 \%$ of respondents had experienced a "near-miss" event while on a procurement trip, and $15 \%$ had been involved in at least one travel-related accident (27). These findings were subsequently supported by a study conducted by a separate team in 2019, in which $23 \%$ of surveyed transplant surgeons reported at least one travel accident with bodily injury (28). These risks may be due to the urgent nature of organ procurement, which often requires travel during adverse weather conditions and may result in poor compliance of safety precautions. The results of these studies merit ongoing consideration of the efficiency and safety of procurement travel.

In addition to the safety hazards, there are striking financial implications associated with organ procurement. A report released by the U.S. Government Accountability Office outlined the staggering rise in costs for ground and air ambulance transportation, billing an average $\$ 40,000$ per ride which is usually an out-of-network expense to patients (29). With the increase in distant travel required for organ retrieval after the recent allocation change, the median cost of organ procurement has more than doubled (from $\$ 34,000$ to $\$ 70,203$ ) and there has been an increased number of "negative" fly-outs, which can cost up to $\$ 15,000$ each (26).

Although published data remains inconsistent regarding the effect of working hours on survival outcomes after transplant surgery, reducing nighttime operations appears to benefit both recipients and surgeons in other regards. Daytime transplant operations have been associated with shorter operative times and decreased blood loss (30). In addition, reducing nighttime operating has been shown to decrease the rates of medical errors, burnout, and 
depression (31). Although further research is required to determine the potential impact, if any, of overnight organ procurement on recipient outcomes, strategies to increase daytime procurement may enhance the availability of medical and technical resources and improve surgeon emotional and mental well-being.

To mitigate many of the obstacles encountered during donor procurement, organization of local transplant teams should be considered. Local procurement teams would allow for timely retrieval of donor organs, while protecting surgeons from long distance travel. Donor lungs could be assessed quickly and effectively, and subsequent transport of the procured organs could proceed without the aid of transplant professionals, preserving the working hours of transplant personnel.

\section{Barriers to increasing the donor pool}

There is a wide discrepancy between reported public support for donation and the availability of organ donors. In a national survey by the U.S. Department of Health and Human Services in 2012, attitudes about organ donation were overwhelmingly positive, with $95 \%$ of adults reporting support for organ donation (32). However, this differs from clinical observation, with only half of these families ultimately agreeing to organ donation $(33,34)$. If a family member objects to a patient's previously documented wishes, what is the correct legal action for organ donation? In 1968, the Uniform Anatomical Gift Act was signed, establishing that a signed donation card is legally sufficient to proceed with organ donation. In the U.S, it is customary for an OPO to obtain permission from the next-of-kin regardless of the documented donor wishes. This is partially due to fear of litigation, but also has been shown to be associated with higher rates of consent (35).

Consent practices vary widely between OPOs. A study by Wendler found that $11 \%$ of OPOs ranked the priority of the deceased's wishes as the most important factor, while $50 \%$ reported family member wishes as the most important factor (36). Many organizations do not have a standard consent policy. To overcome this dilemma, many states have passed "first-person consent" legislation, whereby a patient with documented desire to be an organ donor cannot be overridden by family member wishes. However, studies have shown that adoption of this legislation ultimately has no effect on organ donation (37). Some European countries have enacted "presumed consent" legislation, in which everyone is considered an organ donor unless he or she registers opposition. This contrasts with the current U.S. system of optional consent (or "opt-in" policy), in which the individual or next-of-kin must give explicit consent for organ donation, and presumed consent has been shown to be associated with higher donation rates than countries with optional consent (38). A compelling analysis by Johnson and Goldstein argues this may not be due to the public's fixed attitudes and opinions, but instead may be the influence of how questions regarding donation are asked (39). Standardization of the OPO consent process should be considered to reduce the divergence in practices and improve procurement rates.

Cultural and religious barriers also play a significant role in organ availability. A systematic review investigating the cultural impact on organ donation in the United States found that Asian Americans had the lowest donation rates. The review provided several potential causes for this finding, including reported mistrust of health care, cultural avoidance of discussions regarding death, and maintaining the Confucian principle of filial piety by burying the body intact (40). Although no religion formally forbids organ transplantation, various religious practices preclude the possibility of deceased organ donation. For example, the Jewish faith emphasizes the importance of avoiding interference with the body after death and requires burial of the complete body, preferably within 24 hours. Shinto, the indigenous spirituality of Japan, prohibits interference with a body after death due to its impurity and potential for cursing the living with bad luck (41). These barriers to donation may potentially be addressed with establishment of a donation consent protocol, which could include information responding to common cultural and religious concerns such as public statements on organ donation from religious leaders.

Successful transplant programs in other countries provide valuable lessons for improvement. According to 2018 data published by the Global Observatory on Donation and Transplantation, two countries currently surpass the United States in rates of organ utilization: Spain and Croatia. Spain's success has been attributed to a benchmark study identifying the best-performing hospitals in the country, and subsequent development of protocols for ICU physician teams initiating end-of-life discussions and introducing the option of organ donation (42). Croatia's steadily growing donation rate results purely from deceased donors, as living donation is strongly discouraged due to bioethical principles, with success attributed to establishment of hospital transplant coordinators, public awareness 
campaigns, and donor hospital financial reimbursement (43). For lung transplant donations specifically, the International Registry of Organ Donation (IRODaT) database ranks the U.S. as $7^{\text {th }}$, with the highest rates observed in Austria and Belgium. Further exploration and selective implementation of international strategies may provide innovative solutions to aid in further expanding the donor pool.

\section{Conclusions}

Given the current climate in an ever-expanding demand and critical shortage of donor lungs, harnessing opportunities for improvement in donor assessment, management, and availability is paramount. Additional research must aim to delineate which donor lung characteristics significantly impact recipient survival in order to facilitate availability of donor organs and to identify methods for accurately assessing distant donor organs. To reduce the rising costs and safety risks with distant organ procurement, transplant centers should consider utilization of local procurement teams or establishment of specialized donor care facilities. Also, standardization of the consent process should be pursued in order to improve procurement rates and address the potential cultural and religious barriers to organ donation.

\section{Acknowledgments}

Funding: None.

\section{Footnote}

Provenance and Peer Review: This article was commissioned by the Guest Editor (Jonathan D'Cunha) for the series "Lung Transplantation: Past, Present, and Future" published in Fournal of Thoracic Disease. The article has undergone external peer review.

Conflicts of Interest: All authors have completed the ICMJE uniform disclosure form (available at http:// dx.doi. org/10.21037/jtd-2021-08). The series "Lung Transplantation: Past, Present, and Future" was commissioned by the editorial office without any funding or sponsorship. DK serves as an unpaid editorial board member of fournal of Thoracic Disease. DK reports grants and personal fees from Compass Therapeutics, outside the submitted work. In addition, DK has a pending US patent entitled "Compositions and methods for detecting CCR2 receptors" (application number
15/611,577). pending. VP reports grants from National Institutes of Health, during the conduct of the study; other from Intuitive Surgical, outside the submitted work. All authors have no other conflicts of interest to declare.

Ethical Statement: All authors are accountable for all aspects of the work in ensuring that questions related to the accuracy or integrity of any part of the work are appropriately investigated and resolved.

Open Access Statement: This is an Open Access article distributed in accordance with the Creative Commons Attribution-NonCommercial-NoDerivs 4.0 International License (CC BY-NC-ND 4.0), which permits the noncommercial replication and distribution of the article with the strict proviso that no changes or edits are made and the original work is properly cited (including links to both the formal publication through the relevant DOI and the license). See: https://creativecommons.org/licenses/by-nc-nd/4.0/.

\section{References}

1. Valapour M, Lehr CJ, Skeans MA, et al. OPTN/SRTR 2018 Annual Data Report: Lung. Am J Transplant 2020;20:427-508.

2. Van Raemdonck D, Neyrinck A, Verleden G, et al. Lung donor selection and management. Proc Am Thorac Soc 2009;6:28-38.

3. Egan TM, Kaiser LR, Cooper JD. Lung Transplantation. Curr Probl Surg 1989;26:673-751.

4. Kotecha S, Hobson J, Fuller J, et al. Continued Successful Evolution of Extended Criteria Donor Lungs for Transplantation. Ann Thorac Surg 2017;104:1702-9.

5. Grossman RF, Frost A, Zamel N, et al. Results of SingleLung Transplantation for Bilateral Pulmonary Fibrosis 1990;322:727-33.

6. Singh E, Schecter M, Towe C, et al. Sequence of Refusals for Donor Quality, Organ Utilization, and Survival after Lung Transplantation. J Heart Lung Transplant 2019;38:35-42.

7. Chaney J, Suzuki Y, Cantu E, et al. Lung Donor Selection Criteria. J Thorac Dis 2014;6:1032-8.

8. Chang SH, Kreisel D, Marklin G, et al. Lung Focused Resuscitation at a Specialized Donor Care Facility Improves Lung Procurement Rates. Ann Thorac Surg 2018;105:1531-6.

9. Sommer W, Kuhn C, Tudorache I, et al. Extended criteria donor lungs and clinical outcome: results of an 
alternative allocation algorithm. J Heart Lung Transplant 2013;32:1065-72.

10. Sommer W, Ius F, Muller C, et al. Extended criteria donor lungs do not impact recipient outcomes in pediatric transplantation. J Heart Lung Transplant 2019;38:560-9.

11. Mulligan MJ, Sanchez P, Evans C, et al. The use of extended criteria donors decreases one-year survival in high-risk lung recipients: A review of the United Network of Organ Sharing Database. J Thorac Cardiovasc Surg 2016;152:891-8.e2.

12. Reyes KG, Mason D, Thuita L, et al. Guidelines for donor lung selection: time for revision? Ann Thorac Surg 2010;89:1756-64.

13. Zafar F, Khan M, Heinle J, et al. Does donor arterial partial pressure of oxygen affect outcomes after lung transplantation? A review of more than 12,000 lung transplants. J Thorac Cardiovasc Surg 2012;143:919-25.

14. Fenelon A, Chen LH, Baker SP. Major Causes of Injury Death and the Life Expectancy Gap Between the United States and Other High-Income Countries. JAMA 2016;315:609-11.

15. Hedegaard H, Miniño AM, Warner M. Drug Overdose Deaths in the United States, 1999-2017. NCHS Data Brief 2018;(329):1-8.

16. Goldberg DS, Blumberg E, McCauley M, et al. Improving Organ Utilization to Help Overcome the Tragedies of the Opioid Epidemic. Am J Transplant 2016;16:2836-41.

17. Durand CM, Bowring, MG, Thomas AG, et al. The Drug Overdose Epidemic and Deceased-Donor Transplantation in the United States: A National Registry Study. Ann Intern Med 2018;168:702-11.

18. Sibulesky L, Javed I, Reyes JD, et al. Changing the paradigm of organ utilization from PHS increasedrisk donors: an opportunity whose time has come? Clin Transplant 2015;29:724-7.

19. Phillips KG, Ranganath NK, Malas J, et al. Impact of the Opioid Epidemic on Heart Transplantation: Donor Characteristics and Organ Discard. Ann Thorac Surg 2019;108:1133-9.

20. Gauthier JM, Bierhals AJ, Liu J, et al. Chest computed tomography imaging improves potential lung donor assessment. J Thorac Cardiovasc Surg 2019;157:1711-8.e1.

21. Eberlein M, Reed RM, Bolukbas S, et al. Lung size mismatch and primary graft dysfunction after bilateral lung transplantation. J Heart Lung Transplant 2015;34:233-40.

22. Eberlein M, Permutt $S$, Chahla MF, et al. Lung size mismatch in bilateral lung transplantation is associated with allograft function and bronchiolitis obliterans syndrome. Chest 2012;141:451-60.

23. Doyle MB, Vachharajani N, Wellen JR, et al. A novel organ donor facility: a decade of experience with liver donors. Am J Transplant 2014;14:615-20.

24. Doyle M, Subramanian V, Vachharajani N, et al. Organ Donor Recovery Performed at an Organ Procurement Organization-Based Facility Is an Effective Way to Minimize Organ Recovery Costs and Increase Organ Yield. J Am Coll Surg 2016;222:591-600.

25. Marsolais P, Durand P, Charbonney E, et al. The First 2 Years of Activity of a Specialized Organ Procurement Center: Report of an Innovative Approach to Improve Organ Donation. Am J Transplant 2017;17:1613-9.

26. Puri V, Hachem RR, Frye CC, et al. Unintended Consequences of Changes to Lung Allocation Policy. Am J Transplant 2019;19:2164-7.

27. Englesbe MJ, Merion RM. The Riskiest Job in Medicine: Transplant Surgeons and Organ Procurement Travel. Am J Transplant 2009;9:2406 15.

28. Schenk AD, Washburn WK, Adams AB. A Survey of Current Procurement Travel Practices, Accident Frequency, and Perceptions of Safety. Transplant Direct 2019;5:e494.

29. Air Ambulance: Available Data Show Privately Insured Patients Are at Financial Risk. US Government Accountability Office 2018:1-32.

30. Lindemann J, Dageforde LA, Brockmeier D, et al. Organ procurement center allows for daytime liver transplantation with less resource utilization: May address burnout, pipeline, and safety for field of transplantation. Am J Transplant 2019;19:1296-304.

31. Hendrikx J, Van Raemdonck D, Pirenne J, et al. Outcome of transplantation performed outside the regular working hours: A systematic review and meta-analysis of the literature. Transplant Rev (Orlando) 2018;32:168-77.

32. U.S. Department of Health and Human Services, Health Resources and Services Administration, Healthcare Systems Bureau, 2012 National Survey of Organ Donation Attitudes and Behaviors.

33. Brown CV, Foulkrod K, Dworaczyk S, et al. Barriers to obtaining family consent for potential organ donors. J Trauma 2010;68:447-51.

34. Ebadat A, Brown C, Ali S, et al. Improving organ donation rates by modifying the family approach process. J Trauma Acute Care Surg 2014;76:1473-5.

35. Hanto DW. Family Disagreement over Organ Donation. Virtual Mentor 2005;7:581-3. 
36. Wendler D, Dickert N. The consent process for cadaveric organ procurement: how does it work? How can it be improved? JAMA 2001;285:329-33.

37. Callison K, Levin A. Donor registries, first-person consent legislation, and the supply of deceased organ donors. J Health Econ 2016;49:70-5.

38. Gundle K. Presumed consent: an international comparison and possibilities for change in the United States. Camb Q Healthc Ethics 2005;14:113-8.

39. Johnson EJ, Goldstein D. Defaults and donation decisions. Transplantation 2004;78:1713-6.

40. Li MT, Hillyer GC, Husain SA, et al. Cultural barriers to

Cite this article as: Shepherd HM, Gauthier JM, Puri V, Kreisel D, Nava RG. Advanced considerations in organ donors. J Thorac Dis 2021;13(11):6528-6535. doi: 10.21037/jtd-2021-08 organ donation among Chinese and Korean individuals in the United States: a systematic review. Transpl Int 2019;32:1001-18.

41. Oliver M, Woywodt A, Ahmed A, et al. Organ donation, transplantation and religion. Nephrol Dial Transplant 2011;26:437-44.

42. Matesanz R, Domínguez-Gil B, Coll E, et al. How Spain Reached 40 Deceased Organ Donors per Million Population. Am J Transplant 2017;17:1447-54.

43. Živčić-Ćosić S, Bušić M, Župan Ž, et al. Development of the Croatian model of organ donation and transplantation. Croat Med J 2013;54:65-70. 